\title{
BSE researchers bemoan 'ministry secrecy'
}

Paris. Scientific understanding of the epidemic of bovine spongiform encephalopathy (BSE) in British cattle has been delayed by the reluctance of the UK Ministry of Agriculture, Fisheries and Food (MAFF) to provide access to data, according to UK scientists. This reluctance, they say, has stemmed both from a "culture of secrecy' at the ministry, and its failure to dedicate sufficient staff to analysing and distributing data on the epidemic.

Direct evidence of secrecy at MAFF comes from the uphill struggle faced by Roy Anderson, professor of zoology at the University of Oxford, and his colleagues, in gaining access to the confidential MAFF statistics needed to produce their recent analysis of the transmission dynamics and epidemiology of the BSE epidemic (see Nature 382, 787; 1996).

Indeed, Nature has learnt that MAFF agreed to make the statistics available only after senior officials at the Royal Society put pressure on government ministers, arguing that a credible analysis of MAFF data could be done only by independent experts.

Anderson's study was designed to estimate the number of infected cattle that may have entered the food chain undetected because they were slaughtered before showing clinical symptoms of BSE, and the efficiency of various culling policies designed to reduce the incidence of BSE. Both analyses required raw data on individual farms and the demography of herds, which - according to several sources MAFF initially refused to provide.

"It was pretty clear that MAFF were scared about the outcome," says one scientist involved in the lengthy negotiations, suggesting that this was because the data would suggest - as they did - that many more sick animals had entered the food chain than was previously thought. MAFF eventually backed down and released the data, he says, after it had been persuaded that an independent epidemiological analysis of the BSE analysis was needed, given that Britain's European partners would be sceptical if this were done by MAFF, which would be perceived as having a vested interest in the outcome - only in this way would other European countries be convinced that the study had been carried out "scientifically and properly".

Anderson declines to comment on this account of events. And a ministry spokeswoman dismisses allegations that it has been overly secretive, claiming that access to data is permitted within agreed collaborative projects. Requests for data that are readily available are met automatically, she says. Those for data that are more difficult to compile must be made through a more formal written procedure that includes a fee calculated on the basis of the amount and type of data required, and a pro rata charge for the salary costs needed to prepare it.

The spokeswoman last week promised that scientists can obtain all the data they need "as long as we deem it possible, and as long as the data doesn't infringe on the confidentiality of individual farmers or the data protection act". She declined to provide a copy of the detailed procedures for obtaining data, however, on the grounds that they had to be formally applied for through so-called 'open government' channels.

Not everyone has had problems. Several researchers acknowledge that MAFF has been helpful in providing relatively small sets of data. They point out that aggregated information has been made available, while MAFF's Central Veterinary Laboratory (CVL) in Weybridge has regularly published epidemiological studies. Similarly, Heino Diringer, a researcher at the Robert Koch Institute in Berlin, says that while he has never asked MAFF for statistical data, he has not encountered problems in obtaining other information.

But one scientist who has had difficulty obtaining data from MAFF complains of a "culture of confidentiality" among government ministries, and MAFF in particular. "This has worked to the detriment of the understanding [of the BSE epidemic] and dissemination of information in general," he says, pointing out that the health and environment ministries have better track records "in terms of being open and involving scientists from outside government".

"It has been a nightmare to get hold of comprehensive data," says John Kent, a statistician at the University of Leeds, adding that "not having the numbers more easily available has made life difficult". Kent is keen that MAFF should now make widely available the data used in the Anderson study so that they can be critically assessed.
Anderson agrees that this is necessary. But he points out that the database is not his to give, and that scientists must request it from MAFF. Mark Savey, a leading French epidemiologist working on BSE, says he has been given assurances by MAFF scientists that he will be given access to the particular datasets he wants.

Allegations of excessive secrecy within MAFF are confirmed by one MAFF scientist involved in BSE research. He says that while wider access to data on BSE might not have had much practical impact on the handling of the epidemic, MAFF's lack of openness has been "deplorable". "There is a general principle of not wanting to give the data to anybody. But then the political pressure became so great that it had to be given to the Anderson group," he says. "We shouldn't have been able to withhold data."

The MAFF spokeswoman defends the ministry's actions, however, arguing that its BSE database contains confidential information on individual farms, whose release is forbidden by current government policy; such databases must also respect the provisions of data protection legislation.

Anderson agrees that these factors are important. But he says that they are not insurmountable obstacles to the release of data, pointing out that the databases relating to the AIDS epidemic were made available to the entire scientific community "for analysis and interpretation".

Similarly, the spokeswoman argues that the general release of data could be misleading in the wrong hands. This view is supported by several scientists. "You can't make primary data widely available because it's too complicated," says one. "You need to first make a synthesis." Uncontrolled release of data could cause more problems than it solves, he says, through misinterpretation by both scientists and the press.

\section{Hubble reveals shadow of Jupiter's moon}

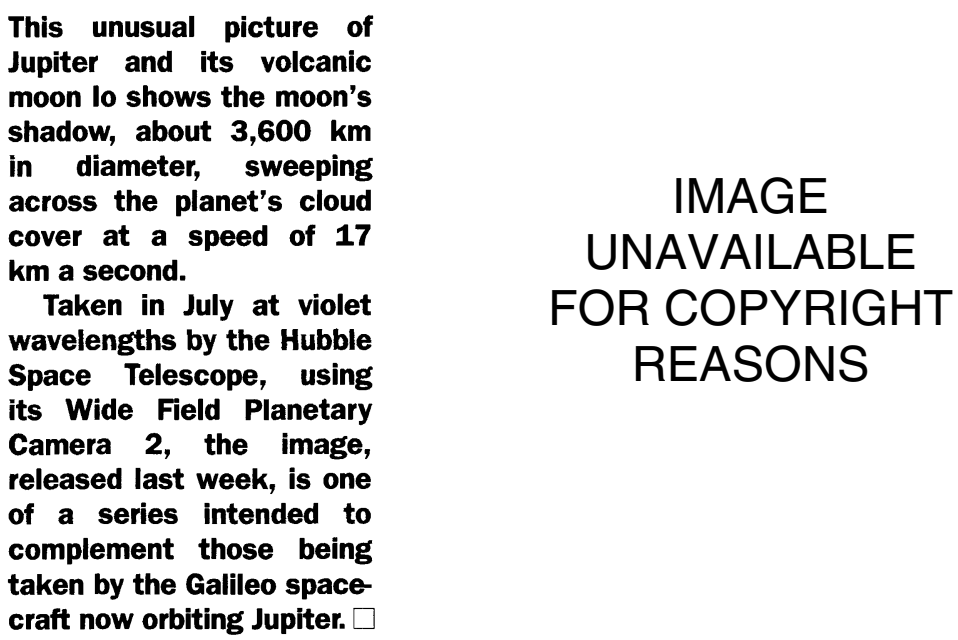


There is general agreement that complex databases should not simply be made freely available, as their proper exploitation requires an understanding of how the data have been assembled, and the various caveats they contain. But many scientists say that analogous problems in other areas such as census data - are overcome by employing individuals to provide such assistance. The CVL would need an extra dozen people to do this, says one MAFF scientist. "It would take a lot of resources, but I think it would be well worth doing."

Indeed, MAFF's failure to assign sufficient staff to the analysis and distribution of BSE data appears to have been a major factor contributing to the difficulties faced by outside scientists. Graham Medley, a biostatistician at the University of Warwick, says he has benefited from greater cooperation from MAFF after having complained on a television programme about the prob-

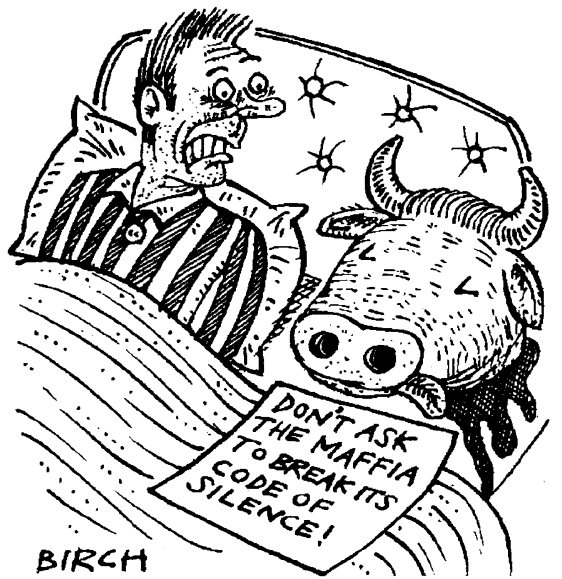

lems of gaining access to MAFF data. Since then he has encountered few such difficulties, and attributes any that have arisen simply to the fact that the CVL is "inundated" with requests, and is "grossly understaffed".

Statistical research on the BSE epidemic within MAFF has been left mainly to a handful of researchers at the CVL. The small numbers of people involved is "staggering for a problem of this importance", says one scientist. Anderson says that, given the scale of the BSE problem, MAFF should have quickly assembled "a large and effective team collaborating with external teams with expertise in particular areas", and that this should have included researchers from other European countries.

Moreover, Anderson argues that cutbacks in government science over the past decade have made such external input more important than ever. These cuts have reduced the government's capacity to carry out the in-house research required to deal with complex scientific issues in many areas, he says. What is now needed, argues Anderson, is an interministerial body - perhaps organized by the Office of Science and Technology - that would assess important scientific issues, decide whether outside expertise was needed, and, if so, arrange for it to be brought in quickly. Declan Butler

\section{New Zealand scientists seek to revive political fortunes}

Sydney. Spending on research by the government of New Zealand has recently begun a slow upturn after a lengthy period of decline. But the scientific and academic communities are still seeking the support of the political parties taking part in the general election on 12 October for further improvements to funding, especially for universities.

Scientists continue to express concern at the effects on research and university teaching of the cost-cutting and restructuring that accompanied the rapid shift towards 'user pays' and 'privatization' or 'corporatization' of public services over the past few years. These policies were introduced by a Labour government and extended by their National Party successors.

But the scientists' campaign is clouded by a new and unpredictable electoral system. Twenty-seven parties are vying for 120 seats under a 'mixed-member proportional' system that has replaced the traditional first-past-the-post method of electing 99 members to the single-chamber parliament.

The New Zealand Association of Scientists (NZAS) and the Royal Society of New Zealand have lobbied for public funding to be restored to the levels that applied when the cuts began 15 years ago. New Zealand spent 1.03 per cent of its gross domestic product on research and development (R\&D) in 1993, placing it fourth from the bottom out of 24 leading economies. Private-sector spending on $R \& D$ is even lower on this list.

Since 1989, responsibility for policy advice to government, funding decisions and the provision of research has been allocated to separate bodies. And scientific merit has been replaced by relevance to national needs as the main criterion for selecting projects to be supported. In 1992, as part of this trend, Simon Upton, the Minister of Research, Science and Technology, abolished the government's Department of Scientific and Industrial Research, replacing it with ten sector-based and commercially oriented Crown Research Institutes (CRIs).

The government justified these changes on the grounds that research performance needed to be more focused in a small country. Claiming last year that the changes have been a success, Upton produced a plan for the next 15 years, which aims to lift government expenditure to 0.8 per cent of gross domestic product.

In particular, Upton has reversed the downward trend of government funding through the new Marsden Fund (NZ\$25 million by next year) for merit-based 'blue skies' research outside the government's priority-setting process and promised that the Public Good Science Fund, which supports projects in CRIs and universities, will be increased and its short-term grants extended.

But many scientists have been disillusioned by the impact of a 30 per cent decline in government funding since 1981. The NZAS has described this decline as "disastrous". A survey of the $\mathbf{3 0 0}$ members of the association in the academic community, government and industry found that the scientific workforce had been "traumatized and decimated" and its productivity "greatly reduced".

According to three past and present officials of the association, writing in the September issue of $N Z$ Science Review, the CRIs have had "varying success, with some functioning well, but others experiencing difficulties, and one small CRI collapsed". Even the effect of the Marsden Fund in sustaining basic knowledge is uncertain, they write.

Despite recent improvements, the survey concludes that morale among scientists is low. "Particularly those in the CRIs were unhappy about their management, which was viewed to be hierarchical, authoritarian, short-term focused and secretive." Job security, it says, has been lost, salaries have declined, time spent on research has decreased, international regard has fallen, and freedom to publish and speak out on policy issues has been restricted.

The governing, conservative National Party has dropped sharply in the opinion polls to 35 per cent. In a recent lecture, Upton promised to boost environmental research through a 'green package' and called for a pause in "tinkering" further with science. Labour, led by Helen Clark, is closing on National with a popular promise to reduce student fees, but is vague about whether it will increase public spending on $R \& D$, claiming it will be interventionist and will not leave investment to the market.

The left-wing Alliance, which promises to abolish student fees altogether, is polling level with NZ First, a party which has now been overtaken by Labour. Its leader, Winston Peters, a populist Maori defector from the Nationals, proposes to "increase public investment in science and technology to the median level of New Zealand's major trading partners" - although without saying where the money will come from.

In higher education, universities, polytechnics and associations of staff and students have joined in a Public Tertiary Education Coalition to oppose the funding cuts planned for the next three years by the National Party.

Peter Pockley 